\title{
Quantum Bourneville Model: On the Quantum Mechanical Nature of Dark Triad
}

\author{
Ajay Agarwal \\ Dept. of Computer Science \& Engineering \\ DIT University \\ Dehradun \\ verslinfiniaudela@gmail.com
}

\begin{abstract}
The state of reality is not bound by the state of progress. Irrespective of our association, or lack of it, with any school of philosophy, the notion that we witness society and the events that occur within it cannot be denied. Consequently, the inception of a significant altercation in the existing direction of any perception regarding an unexplained phenomenon must involve the realization of a requirement for altercation (be it minor or major) to the direction of research conducted in the same. The identification of the correct altercation, hence, becomes the only topic of debate. The process of this identification requires a stoic sense of review of the current state of research and the current state of question that we wish to address. Therefore, it becomes important that this entity must be free from all forms of existing bias and must implement the existing approaches available without the limitations of the subject they are defined in. In this paper, I aim to do the same. This paper presents a thought experiment that eventually paves the way for establishing a quantum mechanical model for interpreting the notion of the Dark Triad whilst addressing all the concerns mentioned in Miller et al. (2019) regarding the current state of research in the field. The frequent question of identification of any empirical proof is rendered moot for this model given its vast intuitive appeal and philosophical foundation. While the author welcomes any attempts possible to provide solid, empirical proof of this quantum Dark Triad model, it is to be noted that the author considers attempts for the same to take into consideration the questions of formalism and determinism as expected from any scientific theory.
\end{abstract}




\section{Current State of Research}

The nature and understanding of "social aversive" or "anti-social" human behavior have piqued the interest of researchers, psychologists, and the general public alike since the word "Dark Triad" came into existence.[12][14]. However, often the abundance of interest in a question doesn't necessarily expedite the discovery of its solution. Similar has been the case for Dark Triads.

Miller et al. (2019) in their recent paper navigated the field of Dark Triad and provide a critical assessment of the current state of research. [27]. Rather unsurprisingly, various concerns regarding the decade-old field have been bought to light. These concerns must be acknowledged by psychologists and academic researcher diligently, to inhibit any future developments in the misconceptions regarding socially-aversive and dark personalities of the society. Despite media glamorization and misinterpretation inflation about the underlying corporate-benefiting traits of narcissism, psychopathy, and Machiavellianism, the academic community must refrain from prioritizing quantitative research over qualitative research. The concerns raised by Miller et al. (2019) in their paper have been summarized in Figure 1. I shall now begin our description of the thought experiment that leads to the formulation of the quantum-mechanics based Dark Triad model.

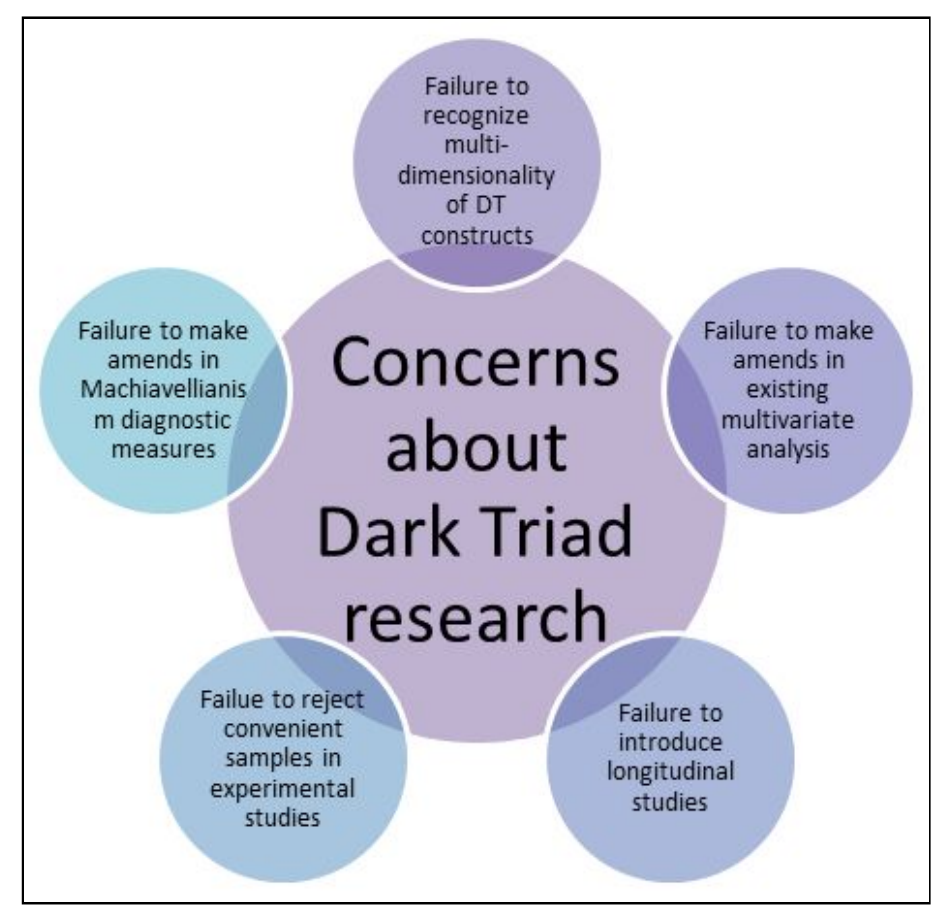

Figure 1. The above figure summarizes the concerns regarding the state-of-research in the Dark Triad field as mentioned by Miller et al. (2019) in their paper.[27]. 


\section{Basics of the Thought Experiment}

The thought experiment begins as following -

Consider a living entity becoming mature with thinking and reasoning capacity when the philosophy of probabilism came into existence. This entity has no upper limit to its measure of age. Over time, this entity witnessed the emergence of the concept of -narcissism, Machiavellianism, and psychopathy. This living and thinking entity also witnessed the time of Einstein and also that of Schrodinger. Finally, as the thinking capacities of this entity grew and strengthened over the period - it came across this paper Miller et al. (2019).[27]. It read the concerns mentioned in this paper and utilizing its existing knowledge of quantum mechanics and probabilism proposed an unbiased solution that utilizes its existing epistemic (knowledge) to understand the ontics (reality) on the DarkTriad. This unbiased approach is not associated with any bias towards any existing model of DarkTriad or any of its components. As this entity is free from any bias, it assigns the same and equal degree of belief to every model ever proposed or published on narcissism, Machiavellianism, and psychopathy.

\section{Philosophical Foundations of the Model}

The entity mentioned in the thought experiment, hence, utilized the philosophy of probabilism to create an unbiased perspective at the current state of research on the identification of the most significant parameter and diagnostic signs for narcissism, Machiavellianism, and psychopathy. This approach is necessary as neither it is humanly possible to filter through all the published research on these fields, whilst making sure no factor mentioned in any model proposed by another person is left out, hence it, in turn, making sure no possible form of bias creeps in.

Probabilism, also known today as - epistemic utility theory. [1-7]. In brief, this theory stems from the philosophy that in the absence of any form of certainty, the probability is the best measure. The entity mentioned in our thought experiment is an academic skeptic and realizes that the feat of total knowledge is impossible, what possible is the association of probability with all existing knowledge (epistemic) to describe the practical affairs, or reality (ontics) of the question at hand (in this case - the question is what diagnostic factors comprise of a practical model that govern the true nature of Dark Triad).

Utilizing the existing knowledge we know about the entity, let's begin formulating this unbiased model more definitively. 
Let $A_{n}$ be a finite set that contains all the factors (represented by $a_{i}$ ) that are proposed in all the existing models on narcissism. Let $\mathrm{A}_{\mathrm{m}}$ be a finite set that contains all the factors (represented by $\mathrm{m}_{\mathrm{i}}$ ) that are proposed in all the existing models on Machiavellianism. Let $\mathrm{A}_{\mathrm{p}}$ be a finite set that contains all the factors (represented by $\mathrm{p}_{\mathrm{i}}$ ) that are proposed in all the existing models on psychopathy. Let $A_{d t}$ be a finite set that contains all the factors (represented by $d_{i}$ ) that are proposed in all the existing models on Dark Triad. Finally, let a finite set of factors denoted by D be defined as -

$$
D=A_{n} \cup A_{m} \cup A_{p} \cup A_{d t}
$$

The set $\mathrm{D}$ contains only the distinct factors that are not duplicated in any other models. Now, according to the epistemic utility theory, let's assign a belief function that stays the same throughout universal time $\mathrm{T}$. Let this universal belief function be denoted an $b_{t}$. As per the rules of epistemic utility theory, this function is defined such that $0 \leq b_{t}(A) \leq 1$, where $\mathrm{A}$ is a proposition. Essentially, the belief function assigns an agent's degree of belief in a proposition at a given time. Now, to make sure no bias creeps in the model that the entity defined in the thought experiment, three main axioms are described.

Axiom 1. $\quad b_{t}\left(a_{i}\right)=x \forall a_{i} \in A_{n}$ where $0 \leq x \leq 1$

This means that every factor that is ever conceptualized as a factor(s) responsible for the diagnosis of narcissism in a person is associated with the same and equal degree of belief.

Axiom 2. $\quad b_{t}\left(m_{i}\right)=x \forall m_{i} \in A_{m}$ where $0 \leq x \leq 1$,

This means that every factor that is ever conceptualized as a factor(s) responsible for the diagnosis of Machiavellianism in a person is associated with the same and equal degree of belief.

Axiom 3: $\quad b_{t}\left(p_{i}\right)=x \forall p_{i} \in A_{p}$ where $0 \leq x \leq 1$,

This means that every factor that is ever conceptualized as a factor(s) responsible for the diagnosis of psychopathy in a person is associated with the same and equal degree of belief.

Axiom 4. $\quad b_{t}\left(d_{i}\right)=x \forall d_{i} \in A_{d t}$ where $0 \leq x \leq 1$,

This means that every factor that is ever conceptualized as a factor(s) responsible for the diagnosis of the Dark Triad in a person is associated with the same and equal degree of belief. 
Axiom 5. The definition of the belief function doesn't with the arrow of time.

This means that irrespective of whether new factors are brought into existence or not in a later time, the definition of the belief function would never change i.e. it shall provide the same value $x$ to every factor in any of the models ever proposed belonging to either of the four sets $A_{n}$ narcissism, $A_{m}$ Machiavellianism, $A_{p}$ psychopathy, and $A_{d t}$ DarkTriad.

This completes our objective formulation of the philosophical background for the model solution proposed by the entity in the thought experiment. I shall now move ahead to provide a quantum mechanical interpretation of the notion of DarkTriad based on the axioms provided as the result of the thought experiment.

\section{Introduction to qDT Model}

With the mathematical establishment of the philosophical foundations out of our way, I shall now describe and explain the quantum mechanical model for Dark Triad utilizing the axioms stated in the previous section. It must be noted that I shall be mostly utilizing the concepts of quantum states, pure states, basis states, quantum superposition, and quantum entanglement for establishing this model. For the audience with no prior background on quantum mechanics, it is advised to refer to this non-technical introduction on quantum mechanics, and the terms required for this paper.[9][15-20]. In either case, irrespective of the reader's choice, the author tries the best to make the qDT model comprehensible at most of the points in this section.

Recall the set $D$. This set contains all the diagnosable factors responsible for "either narcissism or Machiavellianism or psychopathy or DarkTriad". Each factor of this set can be represented as a pure quantum basis state denoted as $\left|\Psi^{\mathrm{s}}{ }_{\mathrm{i}}\right\rangle$, here $s$ represents the corresponding association of the factor with the most immediate diagnosis, i.e., it represents which factor does the set belong to. The value $s$ can take either of the four values $\{n, m, d t, p\}$. Here, $\mathrm{n}$ represents narcissism, $\mathrm{p}$ represents psychopathy, dt represents DarkTriad and $m$ represents Machiavellianism. Hence, the set $D$ can be denoted as -

$$
D=\left\{\Sigma\left|\Psi_{i}^{n}\right\rangle, \Sigma\left|\Psi_{i}^{m}\right\rangle, \Sigma\left|\Psi_{i}^{d t}\right\rangle, \Sigma\left|\Psi_{i}^{p}\right\rangle\right\}
$$


Note - The symbol $\Sigma$ represents the list of all factors with given conditions and not the act of summation. A more succinct representation of set $\mathrm{D}$ would be -

$$
\begin{gathered}
D=\left\{\left|\Psi_{1}{ }^{n}\right\rangle,\left|\Psi_{2}{ }^{n}\right\rangle,\left|\Psi_{3}{ }^{n}\right\rangle \ldots\left|\Psi_{1}{ }^{m}\right\rangle,\left|\Psi_{2}{ }^{m}\right\rangle,\left|\Psi_{3}{ }^{m}\right\rangle \ldots\left|\Psi_{1}^{d t}\right\rangle,\left|\Psi_{2}{ }^{d t}\right\rangle,\left|\Psi_{3}{ }^{d t}\right\rangle\right. \\
\left.\ldots\left|\Psi_{1}{ }^{p}\right\rangle,\left|\Psi_{2}{ }^{p}\right\rangle,\left|\Psi_{3}{ }^{p}\right\rangle \ldots\right\}
\end{gathered}
$$

Let the personality trait narcissism be denoted as pure quantum state $\Psi_{\mathrm{N}}$ which exists as a quantum entangled state (more appropriate would be quantum hyperentangled state to allow for more degrees of freedom [26]) of all quantum states, along with their possible combinations the set D with $s=n$. Then, $\Psi_{\mathrm{N}}$ can be defined as -

$$
\Psi_{\mathrm{N}}=\sum \Psi_{i}^{n}+\sum \Psi_{i}^{n} \Psi_{j}^{n}+\sum \Psi_{i}^{n} \Psi_{j}^{n} \Psi_{k}^{n}+\ldots
$$

The role of these possible combinations is to allow for the creation of n-dimensional sub-models that represent all possible combinations of possible factors responsible for narcissism. Hence, when the narcissism quantum state $\Psi_{\mathrm{N}}$ is measured (definition of 'quantum measurement' is defined later), it shall collapse to one of the basis states - which in our case can also be one of the possible combinations of different factors (which otherwise didn't comprise together as a psychological model in physical reality).

Similar to the narcissism quantum state, two more quantum states $\Psi_{\mathrm{M}}$ and $\Psi_{\mathrm{P}}$ can be created each for Machiavellianism and psychopathy. These quantum states are mathematically defined below-

$$
\begin{gathered}
\Psi_{\mathrm{M}}=\sum \Psi_{i}^{m}+\sum \Psi_{i}{ }^{m} \Psi_{j}{ }^{m}+\sum \Psi_{i}{ }^{m} \Psi_{j}{ }^{m} \Psi_{k}{ }^{m}+\ldots \\
\Psi_{\mathrm{P}}=\sum \Psi_{i}^{p}+\sum \Psi_{i}{ }^{p} \Psi_{j}{ }^{p}+\sum \Psi_{i}{ }^{p} \Psi_{j}{ }^{p} \Psi_{k}{ }^{p}+\ldots
\end{gathered}
$$

I shall now move ahead and begin defining the fourth and last quantum state for the set $\mathrm{A}_{\mathrm{dt}}$. However, unlike our prior definitions, considering possible psychological anomalies, let's first define a new set $\mathrm{D}_{\mathrm{dt}}$. Let the definition of this set be as follows -

$$
D_{d t}=A_{d t}-\left(A_{n} \cup A_{m} \cup A_{p}\right)
$$

This set essentially contains all possible "other" factors that contribute towards the personality trait of Dark Triad, and don't belong to either of the existing three categories - narcissism, Machiavellianism, and psychopathy. This definition stands accurate on our prior formal assumption. Recall our first mention of the definition for the set $A_{d t}$. It is defined as a finite set that contains all the factors that contribute towards DarkTriad. There might likely exist models that propose more than a three-dimensional definition for the concept of DarkTriad and yet might not follow the definitions of the existing three traits. Such factors, by definition, would be an 
element of the set $A_{d t}$. Neglecting them from our model would be inaccurate, and intentionally aligning such factors with the existing definitions of the three personalities would a psychological crime. Hence, I proceed forward in defining a set $A_{d t}$ for such a purpose. From prior knowledge, it would not be an overstatement to say that $\mathrm{D}_{\mathrm{dt}}$ would never be a null set. The proposal of the set $A_{d t}$ and eventually the set $D_{d t}$ allows for consideration of models like DarkTetrad that include the concept of "everyday sadism" as a fourth-dimension.

Following the creation of set $D_{d t}$, the conceptualization and formulation of the quantum state for DarkTriad follows the suit of the previous three. As a different set is defined for DarkTriad factors, I have begun this formulation from the beginning. Let each element (or factor) in the set $D_{d t}$ be defined by a pure basis quantum state $\Phi_{\mathrm{i}}$. Hence, the quantum state for DarkTriad factors, denoted by $\Psi_{\mathrm{dt}}$, is defined as -

$$
\begin{gathered}
\Psi_{d t}=\sum \Phi_{i}+\sum \Phi_{i} \Phi_{j}+\sum \Phi_{i} \Phi_{j} \Phi_{k}+\ldots \\
\text { where } \Phi_{i, j, k, \ldots} \in D_{d t}
\end{gathered}
$$

Finally, I have defined all the possible four dimensions of DarkTriad - narcissism, Machiavellianism, psychopathy, and others (where others are all the factors that don't belong to the first three). So, what is DarkTriad?

Let a pure quantum superposition state exist denoted by $-\Psi_{D T}$. This state, therefore, exists as the quantum superposition of the previous four quantum entangled states -

$$
\Psi_{D T}=c_{1} \Psi_{N}+c_{2} \Psi_{M}+c_{3} \Psi_{P}+c_{4} \Psi_{d t}
$$

where, $c_{i}$ are orthonormal complex numbers i.e., $\left(c_{1}\right)^{2}+\left(c_{2}\right)^{2}+\left(c_{3}\right)^{2}+\left(c_{4}\right)^{2}=1$. What does this mean? The use of quantum superposition at a higher level in our definition construction for DarkTriad helps in preserving the originality of the concept.[24-25]. The concept is, that unlike quantum entangled states that collapse to one or the other basis states on measurement, quantum superposition states are pure and exists continuously together. Upon measurement, the quantum state of DarkTriad $\Psi_{D T}$ instead of collapsing provides an orthonormal distribution of all the four factors that contribute towards the triad, henceforth preserving the originality of the definition for DarkTriad. This summarizes the mathematical formulation of the qDT (quantum Dark Triad) model.

The further sections will deal with questions of the psychological and quantum nature which arise when qDT model is studied from an intense quantum or psychological perspective. 


\section{Quantum Measurement}

Given the quantum mechanical nature of the qDT model, it becomes of immense importance to cautiously decide upon a side for defining "measurement" in the context of this model. The author acknowledges the fact that while there exist seemingly many interpretations that can be applied to the qDT model from a quantum perspective - each with its merit, the choice for a particular interpretation is likely to be influenced by the observer's (reader's) understanding of the qDT model. Analogous to the "measurement problem" in quantum mechanics, the qDT model faces a similar open question - What is the definition of measurement in the qDT model? In this section, for the sake of formulation, I abide by the notion of - "Copenhagen Interpretation" to justify quantum measurement in the qDT model.[21-23].

In brief, Copenhagen Interpretation allows the concept of measurement to be an interference with the outside environment leading to a wave function collapse upon measurement with an apparatus. It also accounts for the vagueness in defining an "accurate measurement apparatus" in classical terms, utilizing the notion of the "Heisenberg cut". It is almost impossible to provide the "most complete" classical specification of a measurement device without fixing the cut (or restricting the observer's ability to move the interface between the classical and quantum in either direction ). Finally, the reader must be aware that the Copenhagen Interpretation is an epistemic theory (i.e., it relies on the existing knowledge of classical and quantum mechanics) and on the question of describing the ontics (reality) utilizing the benefits of "hidden variable" theory (as proposed by Einstein) stands in complete denial.

Extending the notions of Copenhagen Interpretation for the qDT model, I can state the following axioms -

Axiom 6. The concept of measurement in the qDT model is analogous to utilizing the classical diagnostic tools and questionnaires to diagnose the Dark Triad which utilizes a quantitative measure of the scores, ignoring possibilities of inter-variable correlations.

Each time a measurement is taken utilizing any classical apparatus (existing omnibus questionnaires) the quantum state of an agent with DarkTriad collapses into on the basis states due to interaction with the environment (questionnaires). This means that "every existing questionnaire to measure DarkTriad can be computed as "environmental quantum state" that instead of assigning equal possibilities to all three triad traits, assigns some non-equal probabilities for each quantum state". As a result, when a measurement is taken utilizing these apparatus (any questionnaire) the entire quantum state of DarkTriad collapses due to interactions (possibly quantum entanglement) with the environment state. 
Axiom 7. The concept of Heisenberg cut can be stated as the interface between epistemic diagnosis and local diagnosis.

Let this interface be called the "DarkInterface". This means that each questionnaire that exists to measure DarkTriad traits in a person is limited to the constraints of its local knowledge - i.e., it is limited to what the questionnaire 'believes' to be of relevance while diagnosing DarkTriad'. As a result, if the questionnaire in question doesn't include "everyday sadism", for example, as a relevant measure for DarkTriad despite the academic research accepting the same, it is likely the measurement of "everyday sadism" would be missed out from the measurement. Hence, I say that every questionnaire for diagnosing DarkTriad is limited by its local knowledge and results only in what is called a local diagnosis. An epistemic diagnosis, however, would involve utilizing "an almost imaginary questionnaire" that takes into consideration all possible factors that might contribute to the personality traits of DarkTriad. While this would nearly be an impossible feat considering the human limitations, epistemic diagnosis can be achieved utilizing an $n$-criteria questionnaire where $n$ represents the cardinality of the set $D$ defined earlier.

This essentially means that whenever a measurement is taken by any apparatus (questionnaire etc.) the quantum state of DarkTriad for an agent undergoes an irreversible change. Hence, even if many successful measurements are taken in succession utilizing different apparatus, the original quantum state would never be restored, hence the original image of reality (ontic) is lost forever.

To summarize the analogous juxtaposition between the Copenhagen Interpretation on quantum measurement and that of the qDT model, Figure 2 has been utilized for the same. 


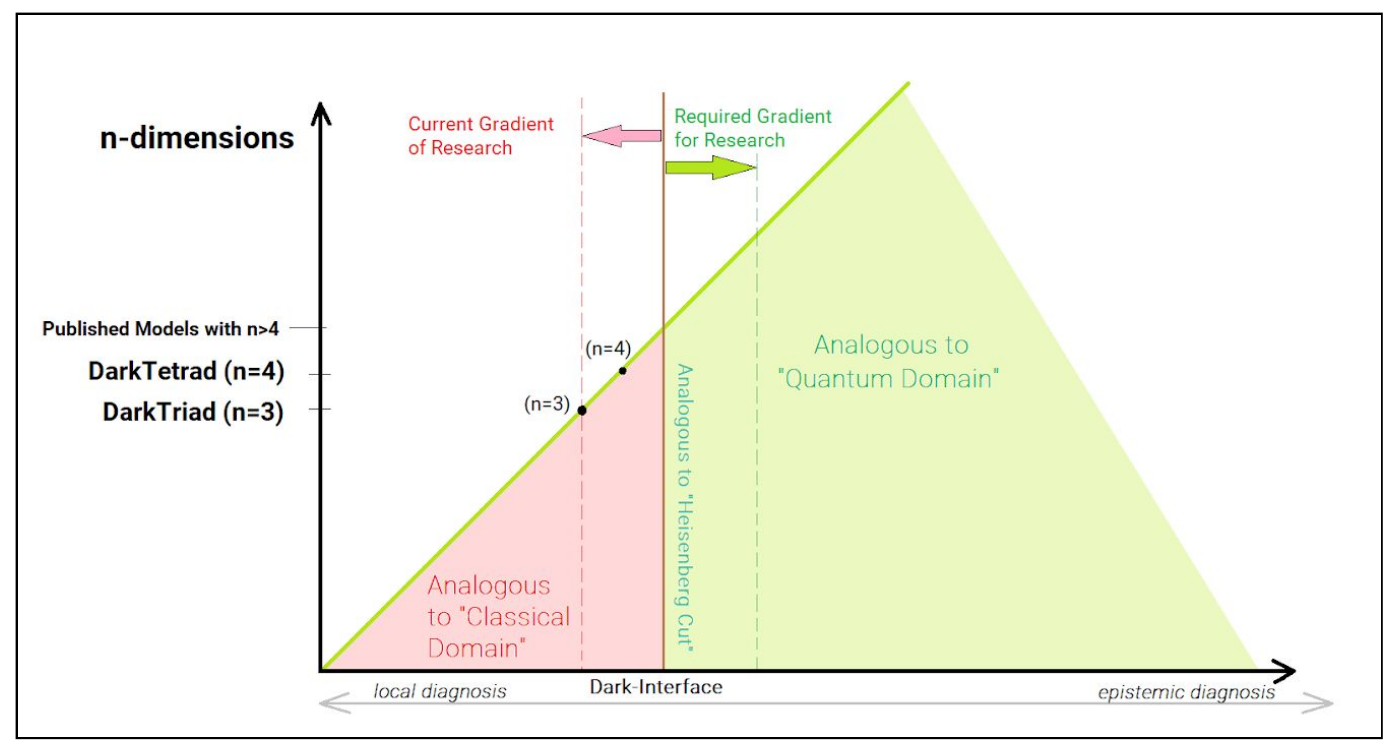

Figure 2. The above figure depicts how the qDT model is analogous to the Copenhagen Interpretation. The figure depicts how the DarkInterface divides local diagnosis from epistemic diagnosis, and how the constant pull of the academic research can lean our understanding of

Dark Triads to parsimonious graves.

\section{Future Considerations and Improvements}

This particular section of the paper would deal with questions that are yet to be addressed and their possible solutions regarding a more intensive quantum mechanical and psychological analysis of the qDT model.

Quantum Questions with no Psychological Answers - One of the main merits of the qDT model is the introduction of the quantum mechanical domain in the psychological space, especially without interfering with the existing state of affairs. However, given the abstract nature of various quantum mechanical concepts, finding the psychological answers for the same becomes a topic for debate.

There exist various untouched questions from quantum mechanics that are yet to be provided answers from the psychological viewpoint. These include -

- What role do quantum gates play in the DarkTriad quantum state? Quantum gates are Pauli matrices that lead to a reversible change in a quantum state. In the case of the qDT model, these correspond to reversible changes brought to the quantum entangled states of the narcissism $\Psi_{\mathrm{N}}$, Machiavellianism $\Psi_{\mathrm{M}}$, psychopathy $\Psi_{\mathrm{P}}$ and DarkTriad $\Psi_{\mathrm{DT}}$. 
- No-cloning theorem - The no-cloning theorem explicitly states that it is impossible to create an exact "duplicate" copy of an unknown quantum state, as long as the state is separable (i.e., the state is not in quantum entanglement.) Now, recall our definition of narcissism, Machiavellianism, and other remaining factors. All these factors were taken as n-state quantum entangled. As the no-cloning theorem doesn't stand applicable to the quantum entangled state, it must be noted that it might be possible to create a duplicate quantum state for the four factors for another agent. Let's say for an agent A - narcissism, Machiavellianism, psychopathy, and DarkTriad is a result of quantum entangled states $\Psi_{\mathrm{N}}^{\mathrm{A}}, \Psi_{\mathrm{M}}^{\mathrm{A}}, \Psi_{\mathrm{P}}^{\mathrm{A}}$ and $\Psi_{\mathrm{DT}}^{\mathrm{A}}$. These quantum entangled states would collapse on measurement to a collection of the basis states responsible for these respective states for the agent A. As no-cloning theorem doesn't stand applicable for entangled states, there exists a non-zero probability that for another agent $\mathrm{B}$ in the world, the factors responsible for the DarkTriad and other traits are the same as that for agent A. However, this possibility could only be explored if an epistemic diagnosis is carried out with an n-criteria questionnaire. As a consequence, for a distant future, until such a questionnaire is created, due to the limitations of the existing local diagnosis, it might appear to the observer's world that no-cloning is possible in the qDT model.

- The question of mathematical visualization - There are several other questions that the qDT model has yet to address. These include a more formal description of corresponding Hilbert spaces in the qDT model [13], quantum teleportation [10] and the possibility of creating a qDT Bloch sphere.[11].

While there might exist more quantum questions that must be addressed by the qDT model, for the introduction of the qDT model in this paper, the above interrogations suffice the cause. I shall now move ahead in trying to list and possibly address questions from the field of psychology (in particular to DarkTriad) which are less likely to be answered from the pens of quantum mechanics.[36-42].

- Practical possibility for an "Epistemic Diagnosis" - This paper introduced the concept of an epistemic diagnosis, rather than an ontic diagnosis. The replacement of epistemic diagnosis with an ontic diagnosis would create a contradiction of the qDT model's alignment with Copenhagen Interpretation. Essentially, an ontic diagnosis means that in "reality" there might exist some "hidden variables" that prevent the qDT model from creating a more deterministic model for DarkTriad and removing the concepts of simultaneously existing probabilities for the factors that contribute to the same. The quantum analogous to this idea is the hidden variable theory, largely attributed to Einstein, which also acted as a major argument as the Copenhagen Interpretation. As stated earlier in the section on Quantum Measurement, the choice of the quantum interpretation to apply with the qDT model can drastically alter the reader's understanding of the qDT model. 


\section{Possibilities of De-labeling}

Finally, I address the lasting "elephant in the room" - the problem of labeling.[8][28-35]. Numerous studies over time have shown with significant confidence that the effect of labeling behaviors traits and even disorders have more damaging and permanent psychological manifestations than advantages as most psychologists presume. Whilst the necessity of identification for different traits, behaviors, and disorders is necessary it is equally important to realize that these labels at least don't stand coded on any grounds which charge undesirable costs from our pursuit for increasing mental health awareness in society. Similar concerns have been raised for the field of DarkTriad as well, especially given the fact that the word "dark" in its most literal sense has negative sentiment and the trait labels already are complicated and at the least have significant universal negative connotations.

For addressing this problem, I would like to suggest the use of "food items" and "places" for the same. An alternative name for "Dark Triad" hence can be the following -

\section{Bourneville Triad}

2. Bourbon Triad

3. Brandy Triad

4. Whisky Triad

5. Cocoa Triad

The possible connations with food items are either their flavor profile or color profile. In the case of the chosen words like Bourneville (as in chocolate brand), Bourbon (biscuits), Brandy (alcohol), Whisky (alcohol), and Cocoa (coffee beans), the color profile for most of these items is at least "dark brown" - which can help psychologist associate the transition from "DarkTriad as malevolent, dark personalities to food items which look dark in color". Despite their dark color profiles, these food items are respectable in society and don't possess a universal flavor profile hence reducing any form of possible misinterpretation leading to stigma, discrimination, or fear.

Based on the possibility of exploring labeling on the criteria of "places", the following names are possible for "DarkTriad" -
1. Croydon Triads
2. Florence Triads
3. Augusta Triads
4. Worcester Triads
5. Calgary Triads 
The places Croydon, Florence, Augusta, Worcester, and Calgary are chosen based on the "birthplace of Havelock Ellis (a psychologist who first introduced the concept of "narcissism")", "birthplace of Niccolo Machiavelli - the ruler to whom the trait "Machiavellianism" is often misattributed to", "birthplace of Hervey Cleckley - the Americal psychiatrist who first introduced the diagnostic criteria for psychopathy in DSM", "birthplace of George Patridge prominent researcher on psychopathy" and "birthplace of Robert Hare - another prominent researcher on psychopathy" respectively. As a result, one might observe it is highly less likely that any sort of negative or stigmatic connotations can be associated with these random places as the history behind these places is likely to be known to a person from the field of psychology. Successful examples of the "place-related coding" are the famous Geneva Trials and the utilized Copenhagen Interpretation.

As the qDT model proposed, over time, maybe the word "triad" can be dropped to introduce the multi-dimensionality of these traits. however, more so than the importance of losing the "triad" word, is the urgency of losing the word "dark".

To the untrained eye, these suggestions might sound absurdist and quite comical, however, it is important to realize that given the severity of this issue and the constant prevalence of ignorance over the matter, it is likely that solutions with the most "out-of-the-box" approaches and oddest first perceptions are more likely to stand the test of time and eventually emerge correctly on a thoughtful and insightful introspection. The reader must also note that as the COVID19 pandemic has regained society's attention and successfully diverted the same towards realizing the importance of mental health, it becomes crucial that psychologist and psychiatrists in the field make sure to redeem their lost image NOT as a deeply complex, divided, and a stigmatically labeled field BUT as a field of immense importance, severity and universal acceptance with almost no scope of bias and discrimination.

About this paper, the author can safely say that to introduce de-labeling in the author's future research work (and for the field in general), the qDT (quantum Dark Triad) model can be renamed as the Quantum Bourneville Model. 


\section{Conclusion}

In this paper, I present to the readers the qDT model (Quantum Dark Triad) model, now known as Quantum Bourneville Model. Whilst a ton of research has been conducted and is being conducted on the Dark Triad trait of personality, the Quantum Bourneville Model is one-of-its-kind, for that it provides not only an approach to understand a question that is yet to be solved, but also collects together all the approaches utilized till date for solving the question altogether allowing rooms for future approaches.

\section{Declaration, data usage and funding}

The author declares no potential conflict of interest. No data was used, as the paper is a theoretical discourse. No funding was obtained from any institute for the conducted work.

\section{Declaration of competing interest}

The authors declare that they have no known competing financial interests or personal relationships that could have appeared to influence the work reported in this paper. 


\section{References}

1. Marmodoro, A. (2015). Introduction: The Metaphysics of Quantum Mechanics. Topoi, 34(2), 309-311.

2. Feyerabend, P. (2015). Philosophical Problems of Quantum Theory(1964). In S. Gattei \& J. Agassi (Eds.), Physics and Philosophy: Philosophical Papers (pp. 346-365).

Cambridge: Cambridge University Press. doi:10.1017/CBO9781139034197.026

3. De, F. B. (1989). Probabilism: A Critical Essay on the Theory of Probability and on the Value of Science.

4. Cabello, A. (2017). Interpretations of quantum theory: a map of madness. What is quantum information, 138-144.

5. Wang, H., Smith, J. W., \& Sun, Y. (2019). Simulating Cognition with Quantum Computers. arXiv preprint arXiv:1905.12599.

6. Jacobs, K., \& Steck, D. A. (2006). A straightforward introduction to continuous quantum measurement. Contemporary Physics, 47(5), 279-303.

7. Pettigrew, R. (2012). An improper introduction to epistemic utility theory. In EPSA Philosophy of Science: Amsterdam 2009 (pp. 287-301). Springer, Dordrecht.

8. Butera, C. (2020, August 28). A Primer on De-Identifying and Anonymizing Social Psychology Data. https://doi.org/10.31234/osf.io/aujrs

9. Grabbe, J. O. (2005). An introduction to quantum game theory. arXiv preprint quant-ph/0506219.

10. Luo, Y. H., Zhong, H. S., Erhard, M., Wang, X. L., Peng, L. C., Krenn, M., ... \& Zeilinger, A. (2019). Quantum teleportation in high dimensions. Physical review letters, 123(7), 070505.

11. Aerts, D., \& Sassoli de Bianchi, M. (2016). The extended Bloch representation of quantum mechanics: Explaining superposition, interference, and entanglement. Journal of Mathematical Physics, 57(12), 122110.

12. McLarnon, M. J., \& Tarraf, R. C. (2020). Getting to the core: How “(dis) honest" is the core of the Dark Triad?. Personality and Individual Differences, 110545.

13. Liu, J. (1993). Evolution of pure states into mixed states. arXiv preprint hep-th/9301082.

14. Furnham, A., Richards, S. C., \& Paulhus, D. L. (2013). The Dark Triad of personality: A 10 year review. Social and Personality Psychology Compass, 7(3), 199-216.

15. Ball, P. (1999). Everyone wins in quantum games.

16. Piotrowski, E. W., \& Sładkowski, J. (2003). An invitation to quantum game theory. International Journal of Theoretical Physics, 42(5), 1089-1099.

17. Landau, L. (1927). The damping problem in wave mechanics. Journal of Physics , 45 (5-6), 430-441.

18. Landau, L. D. (1965). E. hl. Lifshitz, Quantum mechanics, non-relativistic theory.

19. Dirac, P. A. M. (1958). The principles of quantum mechanics, 4th edn. Clarendon. 
20. Bohr, N. (1927/1928). The quantum postulate and the recent development of atomic theory, Nature Supplement April 14 1928, 121: 580-590.

21. Heisenberg, W. (1985). About the descriptive content of quantum theoretical kinematics and mechanics. In Original Scientific Papers Original Scientific Papers (pp. 478-504). Springer, Berlin, Heidelberg.

22. Einstein, A. (1949). Remarks concerning the essays brought together in this co-operative volume. Albert Einstein: Philosopher-Scientist, 2, 665-688.

23. Pauli, W. E. F., \& Källén, G. (1958). Handbuch der physik.

24. Dabrowski, L. (1991). A superposition principle for mixed states?. Il Nuovo Cimento B (1971-1996), 106(9), 963-968.

25. Passante, G., Emigh, P. J., \& Shaffer, P. S. (2015). Student ability to distinguish between superposition states and mixed states in quantum mechanics. Physical Review Special Topics-Physics Education Research, 11(2), 020135.

26. Stenholm, S., \& Bardroff, P. J. (1998). Teleportation of N-dimensional states. Physical Review A, 58(6), 4373.

27. Miller, J., Vize, C., Crowe, M. L., \& Lynam, D. (2019, February 14). A critical appraisal of the Dark Triad literature and suggestions for moving forward. https://doi.org/10.31234/osf.io/mbkr8

28. Wright, A., Jorm, A. F., \& Mackinnon, A. J. (2011). Labeling of mental disorders and stigma in young people. Social science \& medicine (1982), 73(4), 498-506. https://doi.org/10.1016/j.socscimed.2011.06.015

29. McPherson, S., \& Armstrong, D. (2006). Social determinants of diagnostic labels in depression. Social science \& medicine, 62(1), 50-58.

30. "'Judging a book by its cover': An experimental study of the negative impact of a diagnosis of borderline personality disorder on clinicians' judgements of uncomplicated panic disorder." British Journal of Clinical Psychology. doi: 10.1111/bjc.12093

31. Drapalski, A. L., Lucksted, A., Perrin, P. B., Aakre, J. M., Brown, C. H., DeForge, B. R., \& Boyd, J. E. (2013). A model of internalized stigma and its effects on people with mental illness. Psychiatric Services, 64(3), 264-269.

32. Angermeyer, M. C., \& Dietrich, S. (2006). Public beliefs about and attitudes towards people with mental illness: a review of population studies. Acta Psychiatrica Scandinavica, 113(3), 163-179.

33. Corrigan, P. W., \& Watson, A. C. (2002). Understanding the impact of stigma on people with mental illness. World psychiatry, 1(1), 16.

34. Garand, L., Lingler, J. H., Conner, K. O., \& Dew, M. A. (2009). Diagnostic labels, stigma, and participation in research related to dementia and mild cognitive impairment. Research in gerontological nursing, 2(2), 112-121.

35. Crocker, J., Quinn, D. M., Heatherton, T. F., Kleck, R. E., Hebl, M. R., \& Hull, J. G. (2000). The social psychology of stigma. 
36. Lynam, D. R., \& Miller, J. D. (2015). Psychopathy from a basic trait perspective: The utility of a five-factor model approach. Journal of Personality, 83(6), 611-626.

37. Miller, J. D., Lynam, D. R., Hyatt, C. S., \& Campbell, W. K. (2017). Controversies in narcissism. Annual Review of Clinical Psychology, 13, 291-315.

38. Miller, J. D., Lynam, D. R., McCain, J. L., Few, L. R., Crego, C., Widiger, T. A., \& Campbell, W. K. (2016). Thinking structurally about narcissism: An examination of the Five-Factor Narcissism Inventory and its components. Journal of Personality Disorders, 30(1), 1-18.

39. Krizan, Z., \& Herlache, A. D. (2018). The narcissism spectrum model: A synthetic view of narcissistic personality. Personality and Social Psychology Review, 22(1), 3-31.

40. Miller, J. D., Hyatt, C. S., Maples-Keller, J. L., Carter, N. T., \& Lynam, D. R. (2017). Psychopathy and Machiavellianism: A distinction without a difference? Journal of personality, 85(4), 439-453.

41. Miller, J. D., Dir, A., Gentile, B., Wilson, L., Pryor, L. R., \& Campbell, W. K. (2010). Searching for a vulnerable dark triad: Comparing factor 2 psychopathy, vulnerable narcissism, and borderline personality disorder. Journal of personality, 78(5), 1529-1564.

42. Jammer, M. (1974). Philosophy of Quantum Mechanics. the interpretations of quantum mechanics in historical perspective. 
Figures

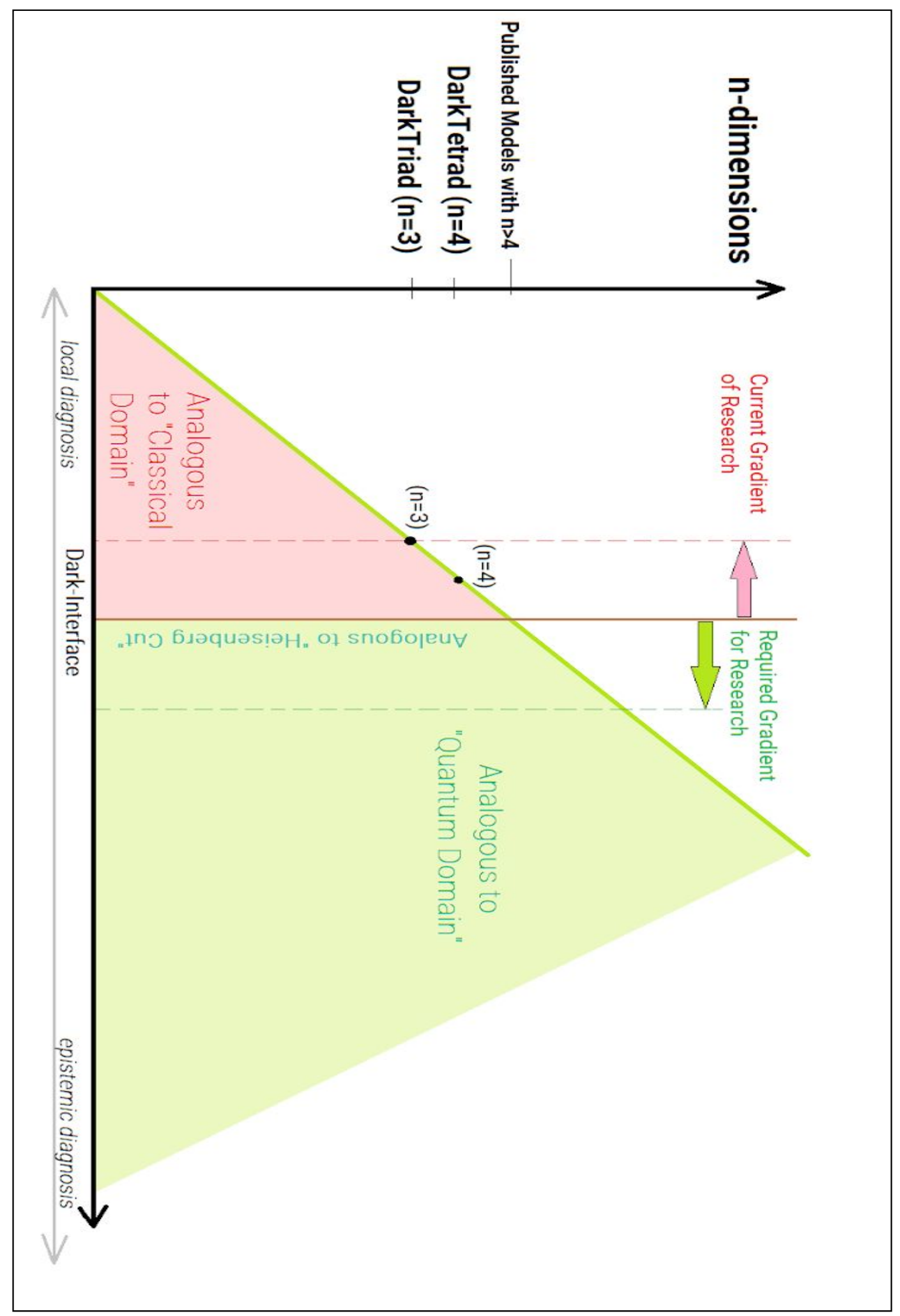

Figure 1 


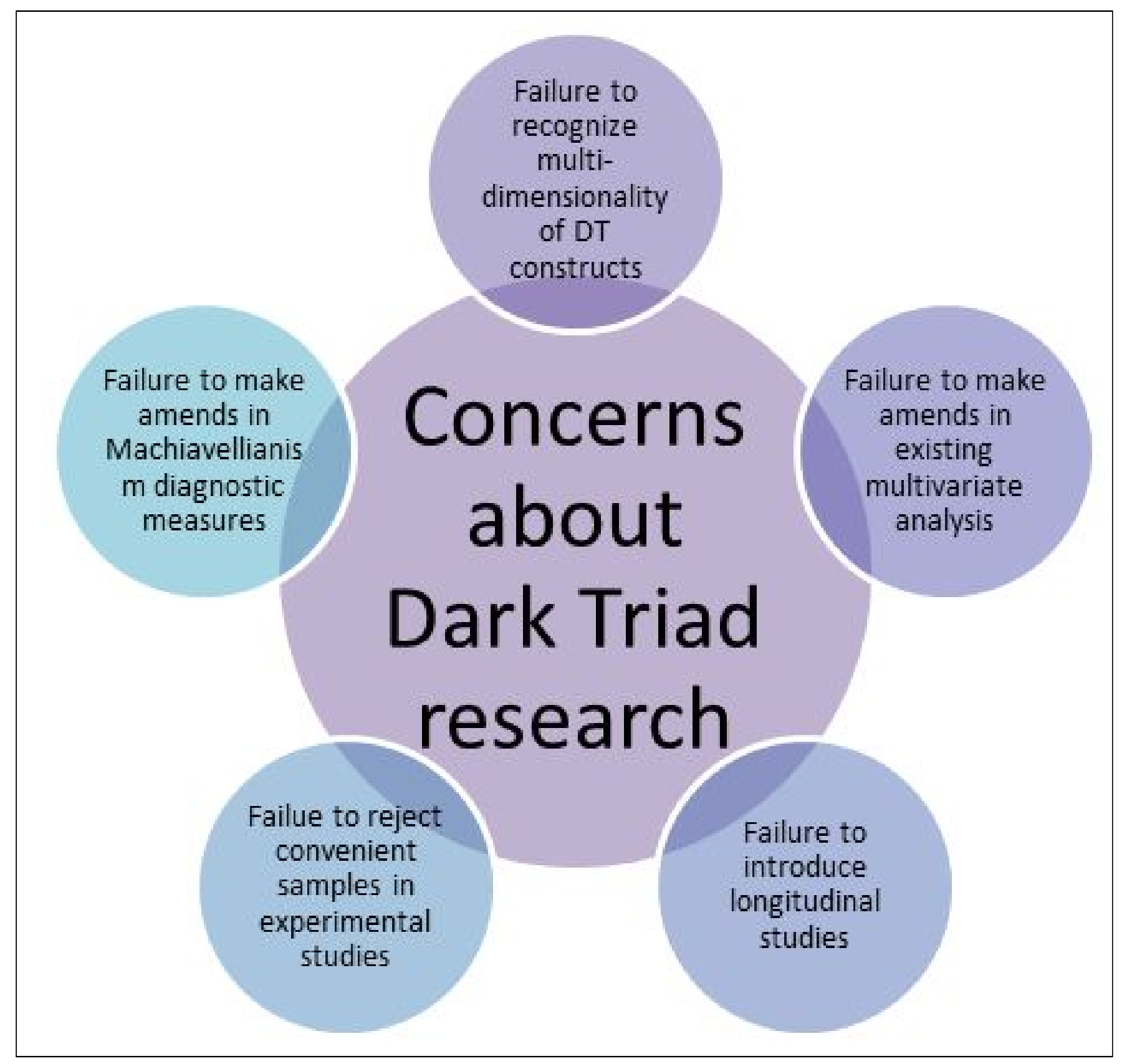

Figure 2 